\title{
Model equations from gyrokinetic theory for a non-neutral plasma to include temperature effects and applications to a plasma of infinite length
}

S. Neil Rasband

judith@conselle.com

Follow this and additional works at: https://scholarsarchive.byu.edu/facpub

Part of the Astrophysics and Astronomy Commons, and the Physics Commons

\section{Original Publication Citation}

Rasband, Neil S."Model equations from gyrokinetic theory for a non-neutral plasma to include temperature effects and applications to a plasma of infinite length." Physics of Plasmas 3 (1996): 94-13.

\section{BYU ScholarsArchive Citation}

Rasband, S. Neil, "Model equations from gyrokinetic theory for a non-neutral plasma to include temperature effects and applications to a plasma of infinite length" (1996). Faculty Publications. 683. https://scholarsarchive.byu.edu/facpub/683 accepted for inclusion in Faculty Publications by an authorized administrator of BYU ScholarsArchive. For more information, please contact ellen_amatangelo@byu.edu. 


\title{
Model equations from gyrokinetic theory for a non-neutral plasma to include temperature effects and applications to a plasma of infinite length
}

\author{
S. Neil Rasband ${ }^{\text {a) }}$ \\ Department of Physics and Astronomy, 180 Eyring Science Center, Brigham Young University, \\ Provo, Utah 84602
}

(Received 8 August 1995; accepted 25 September 1995)

\begin{abstract}
Gyrokinetic equations are derived for applications to non-neutral plasmas in constant, straight magnetic fields wherein $\mathrm{E} \times \mathrm{B}$ drift velocities are of the same order as thermal velocities. The ratio of the $\mathrm{E} \times \mathrm{B}$ rotation frequency to the cyclotron frequency and the ratio of the gyroradius to a plasma scale length are assumed to be of order $\epsilon$ and terms are retained in the gyrokinetic expansion to second order to include finite-Larmor-radius (FLR) effects. A mode equation is obtained for a non-neutral plasma in the infinite-length approximation. The singularities of this equation are compared and contrasted with the familiar mode equation from the cold-fluid approximation. A numerical investigation of $m=1$ perturbations for a pure electron plasma with parameters chosen to closely approximate those in the report by Driscoll [Phys. Rev. Lett. 64, 645 (1990)] reveals no exponentially unstable modes with significant growth rates and strongly suggests that finite temperature is not the source of the exponential growth seen in the experiments. (C) 1996 American Institute of Physics. [S1070-664X(96)02401-7]
\end{abstract}

\section{INTRODUCTION}

In this paper we obtain the gyrokinetic equations suitable for application to a non-neutral plasma. The gyrokinetic expansion is carried out to second order in $\epsilon$, where both the ratio of the $\mathrm{E} \times \mathrm{B}$ rotational frequency to the electron cyclotron frequency and the ratio of the gyroradius to the plasma scale size are considered to be of order $\epsilon$. The gyrokinetic results are applied to a situation where there is no $z$-dependence in either the equilibrium or the perturbation and we study the $m=1$ diocotron mode for hollow profiles.

This study was primarily motivated by experimental observations of an $m=1$, exponentially unstable diocotron mode in a hollow electron column by Driscoll ${ }^{1}$ and the fact that the cause of this instability remains ambiguous. In a conventional two-dimensional (2-D) drift model (no inertia and zero temperature) linear theory predicts that there are no $m=1$ exponentially unstable modes. In a companion article to Driscoll's, Smith and Rosenbluth ${ }^{2}$ describe analytic and numerical investigations of the same system within the 2-D drift model wherein they find algebraic growth of $m=1$ perturbations $\propto \sqrt{t}$. They refer to preliminary calculations that suggest a small gyroradius or viscosity can lead to exponential growth. In a subsequent letter, $\mathrm{Smith}^{3}$ explored in a phenomenological way the effects of finite gyroradius and finite axial length on exponential growth. A study by Rasband et al. ${ }^{4}$ using a warm fluid model suggested that the exponential instability may be due to finite temperature; however, the calculated growth rate was still an order of magnitude below the experimentally measured result and also exhibited oscillations with changing temperature that seem unphysical.

The new results reported here show that the growth rates that result from a careful inclusion of temperature effects via gyrokinetic theory are above those reported by $\mathrm{Smith}^{3}$ but

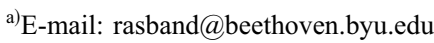

below those reported in Ref. 4 and still cannot explain the observed instability. Specifically, in the circumstance where there is no dependence on the $z$ coordinate, for a hollow profile and a temperature of $1.2 \mathrm{eV}$ (consistent with Ref. 1) there are no modes with growth rates greater than about $10^{-3}$ times the real part of the frequency for the mode.

A second outcome of this work has been a discovery of the way in which the inclusion of kinetic effects modifies the singularities of the mode equation.

\section{THE TRANSFORMATION TO GUIDING CENTER (GC) COORDINATES}

To obtain a gyrokinetic description of a pure electron plasma where the gyrophase has been averaged out in a selfconsistent way, we follow the phase space Lagrangian approach using Lie transforms developed by Littlejohn. ${ }^{5}$ Our calculation, being directed towards a different purpose, differs from those already reported in the literature. First we consider the magnetic field to be straight, aligned along the $z$-axis, externally imposed, and constant in time and space. Second, the self-consistent electric field for the non-neutral plasma is of the same order as the magnetic field. Furthermore, we pull out explicitly the $\mathrm{E} \times \mathrm{B}$ drift velocity as of the same order as the gyromotions. These ordering considerations lead us to take as a particle phase space Lagrangian,

$$
\begin{array}{r}
\int L d t=\int d t\left\{\left(\frac{1}{\boldsymbol{\epsilon}} \frac{M \Omega}{2} \hat{\mathbf{b}} \times \mathbf{x}+M u \hat{\mathbf{b}}+M w \hat{\mathbf{c}}+M \mathbf{v}_{e}\right) \cdot \dot{\mathbf{x}}\right. \\
\left.-\left(\frac{1}{\epsilon} q \Phi+\frac{1}{2} M u^{2}+\frac{1}{2} M w^{2}+\frac{1}{2} M v_{e}^{2}+M w \hat{\mathbf{c}} \cdot \mathbf{v}_{e}\right)\right\},
\end{array}
$$

where $M$ is the mass of the particle in question and $q$ its 
charge; $\mathbf{B}$ is the (imposed) magnetic field; $\hat{\mathbf{b}}=\mathbf{B} /|\mathbf{B}| \equiv \hat{\mathbf{e}}_{3}$ with $\hat{\mathbf{e}}_{1}, \hat{\mathbf{e}}_{2}$ fixed spatial unit vectors, such that $\hat{\mathbf{e}}_{1} \times \hat{\mathbf{e}}_{2}=\hat{\mathbf{e}}_{3} ; \theta$ is the gyrophase angle with $\hat{\mathbf{c}}=\sin \theta \hat{\mathbf{e}}_{1}-\cos \theta \hat{\mathbf{e}}_{2}$ and $\hat{\mathbf{b}} \times \hat{\mathbf{c}}$ $=\hat{\mathbf{a}}=\cos \theta \hat{\mathbf{e}}_{1}+\sin \theta \hat{\mathbf{e}}_{2} ; c$ is the velocity of light and $\Omega=q B / M c$ is the signed gyrofrequency; $\Phi$ is the electrostatic potential and $\mathbf{v}_{e}=(q / M \Omega) \mathbf{E} \times \hat{\mathbf{b}}$. The particle velocity is written as $\mathbf{v}=u \hat{\mathbf{b}}+w \hat{\mathbf{c}}+\mathbf{v}_{e}$. The ordering parameter $\epsilon$ has been introduced explicitly for the usual purpose of tagging the terms of various orders, and is set equal to 1 for obtaining numerical results. In Eq. (1) the electrostatic potential has been given the same order as the vector potential since $\left|\mathbf{v}_{e}\right|$ is of the same order as the velocity space variables $w$ and $u$.

Due to the more-or-less straightforward nature of the Lie transform technique and the fact that several articles containing such gyrokinetic transformations have appeared in this journal and elsewhere, ${ }^{5-11}$ the details of this calculation have been relegated to an appendix. Suffice it to say that it must be carried out to fourth order to fully determine the required coordinate transformation to second order. The guiding center (GC) coordinates are denoted as $(\mathbf{X}, U, \Theta, W)$, where $\mathbf{X}$ denotes the position of the guiding center and $(U, \Theta, W)$ are velocity coordinates with $U$ denoting the parallel velocity and $\Theta$ the gyrophase. The resulting Lagrangian in GC coordinates is

$$
\begin{aligned}
\int L d t= & \int d t\left\{\left[\frac{1}{\epsilon} \frac{M \Omega}{2} \hat{\mathbf{b}} \times \mathbf{X}+M U \hat{\mathbf{b}}+M \mathbf{v}_{e}\right] \cdot \dot{\mathbf{X}}\right. \\
& -\frac{\epsilon M W^{2}}{2 \Omega} \dot{\Theta}-\left[\frac{1}{\epsilon} q \Phi+\frac{1}{2} M\left(U^{2}+W^{2}+v_{e}^{2}\right)\right. \\
& \left.\left.+\epsilon \frac{M W^{2}}{4 \Omega}(\hat{\mathbf{b}} \cdot \boldsymbol{\psi})-\epsilon^{2} \Gamma_{t}^{(3)}+\mathscr{O}\left(\epsilon^{3}\right)\right]\right\},
\end{aligned}
$$

where $\boldsymbol{\psi} \equiv \nabla \times \mathbf{v}_{e}$ and $\Gamma_{t}^{(3)}$ is given by

$$
\begin{aligned}
\Gamma_{t}^{(3)}= & -\frac{M W^{2}}{8 \Omega^{2}}\left[\left(\nabla v_{e}\right)^{2}+\psi_{\perp}^{2}-\frac{3}{2}(\hat{\mathbf{b}} \cdot \boldsymbol{\psi})^{2}\right]+\frac{M}{2 \Omega^{2}}\left[\left(\frac{D \mathbf{v}_{e}}{D t}\right.\right. \\
& \left.\left.+\mathbf{v}_{e} \times \psi_{\perp}\right)^{2}-\left(b v_{e} v_{e}\right)^{2}\right]+\frac{M v_{\|}}{2 \Omega^{2}} \psi \cdot \frac{D \mathbf{v}_{e}}{D t},
\end{aligned}
$$

where

$$
(\nabla \mathbf{v})^{2} \equiv \nabla \mathbf{v}: \nabla \mathbf{v}=v_{i, j} v_{i, j},
$$

and the last form summed over repeated indices is for Cartesian components. For brevity we have used

$$
\frac{D}{D t} \equiv \frac{\partial}{\partial t}+\left(\mathbf{v}_{e}+U \hat{\mathbf{b}}\right) \cdot \nabla .
$$

Note that

$$
\frac{\partial \Gamma_{t}^{(3)}}{\partial U}=\frac{M}{\Omega^{2}} \hat{\mathbf{b}} \cdot\left[\psi \times \frac{D \mathbf{v}_{e}}{D t}\right] .
$$

The Euler-Lagrange equations obtained from (2) in GC coordinates $(\mathbf{X}, U, \Theta, W)$ (collectively denoted by $\left.Z^{\mu}, \mu=1, \ldots, 6\right)$ give

$$
\begin{aligned}
& \dot{\mathbf{X}}=U \hat{\mathbf{b}}+\mathbf{v}_{e}+\frac{\epsilon}{\Omega} \hat{\mathbf{b}} \times \frac{D \mathbf{v}_{e}}{D t}+\frac{\epsilon^{2}}{\Omega^{2}}\left\{\frac{W^{2}}{4} \nabla_{\perp}^{2} \mathbf{v}_{e}\right. \\
& \left.+\boldsymbol{\psi} \times\left[\hat{\mathbf{b}} \times\left(\hat{\mathbf{b}} \times \frac{D \mathbf{v}_{e}}{D t}\right)\right]\right\}+\mathscr{O}\left(\epsilon^{3}\right), \\
& =U \hat{\mathbf{b}}+\left(1+\epsilon^{2} \frac{1}{4} \frac{W^{2}}{\Omega^{2}} \nabla_{\perp}^{2}\right) \mathbf{v}_{e}+\left(\frac{q}{M \Omega}\right) \frac{\epsilon}{\Omega}[1 \\
& \left.+\frac{\epsilon}{\Omega} \boldsymbol{\psi} \times \hat{\mathbf{b}} \times\right] \frac{D \mathbf{E}_{\perp}}{D t}+\mathcal{O}\left(\epsilon^{3}\right), \\
& \mu \equiv-\frac{\epsilon M W^{2}}{2 \Omega}=\text { const, } \\
& \dot{U}=\frac{\Omega}{\epsilon}\left(\frac{q}{M \Omega}\right)(\hat{\mathbf{b}} \cdot \mathbf{E})-\frac{\epsilon}{\Omega}\left[1-\frac{\epsilon}{\Omega}(\hat{\mathbf{b}} \cdot \boldsymbol{\psi})\right]\left(\frac{D \mathbf{v}_{e}}{D t} \cdot \boldsymbol{\psi}\right) \\
& -\frac{\epsilon}{\Omega} \frac{W^{2}}{4} \hat{\mathbf{b}} \cdot \nabla(\hat{\mathbf{b}} \cdot \boldsymbol{\psi})-\frac{\epsilon^{2}}{\Omega^{2}}\left[\frac{W^{2}}{4} \hat{\mathbf{b}} \cdot\left(\boldsymbol{\psi} \times \nabla_{\perp}^{2} \mathbf{v}_{e}\right)\right. \\
& \left.-\frac{\Omega^{2}}{M} \hat{\mathbf{b}} \cdot \nabla \Gamma_{t}^{(3)}\right]+\mathscr{O}\left(\epsilon^{3}\right), \\
& \dot{\Theta}=-\frac{1}{\epsilon} \Omega-\frac{1}{2} \hat{\mathbf{b}} \cdot \boldsymbol{\psi}+\frac{1}{4} \frac{\epsilon}{\Omega}\left[\frac{3}{2}(\hat{\mathbf{b}} \cdot \boldsymbol{\psi})^{2}-\left(\nabla \mathbf{v}_{e}\right)^{2}-\psi_{\perp}^{2}\right] \\
& +\mathscr{Q}\left(\epsilon^{3}\right) \text {. }
\end{aligned}
$$

Equation (8) has been written in such a form that we readily recognize the familiar polarization drift plus corrections and the FLR correction to the $\mathrm{E} \times \mathrm{B}$ drift $\mathbf{v}_{e}$. Equation (7) is more useful for subsequent calculations. By choosing to push all higher order corrections into the Hamiltonian part of the phase space Lagrangian (see Ref. 5), Eq. (9) is exact to all orders.

The Vlasov equation for the distribution function $F$ in $\mathrm{GC}$ coordinates becomes

$$
\frac{\partial F}{\partial t}+\dot{\mathbf{X}} \cdot \nabla F+\dot{U} \frac{\partial F}{\partial U}=0 .
$$

In Eq. (12) no $\dot{W}$ terms appear because $W$ is constant and no $\dot{\Theta}$ terms appear because $F$ is independent of $\Theta$ to the order calculated. For a situation to be considered shortly where there is no dependence on the coordinate $z$ along the $\hat{\mathbf{b}}$ direction, $\dot{U}=0$ as well.

We assume the potential to be an equilibrium potential, plus a perturbation, $\Phi=\Phi^{(0)}+\Phi^{(1)}$, with corresponding density, $n=n^{(0)}+n^{(1)}$, distribution function, $F=F^{(0)}+F^{(1)}$, and drift velocity, $\mathbf{v}_{e}=\mathbf{v}_{e}^{(0)}+\mathbf{v}_{e}^{(1)}$. We linearize in this perturbation and obtain from Eq. (12),

$$
\begin{gathered}
\frac{\partial F^{(0)}}{\partial t}+\dot{\mathbf{X}}^{(0)} \cdot \nabla F^{(0)}+\dot{U}^{(0)} \frac{\partial F^{(0)}}{\partial U}=0, \\
\frac{\partial F^{(1)}}{\partial t}+\dot{\mathbf{X}}^{(0)} \cdot \nabla F^{(1)}+\dot{U}^{(0)} \frac{\partial F^{(1)}}{\partial U} \\
=-\dot{\mathbf{X}}^{(1)} \cdot \nabla F^{(0)}-\dot{U}^{(1)} \frac{\partial F^{(0)}}{\partial U} .
\end{gathered}
$$


Each of these Eqs. (13) and (14) is solved to $\mathscr{Q}\left(\epsilon^{2}\right)$ in the $\mathrm{GC}$ expansion. Linearized in the perturbation, Eq. (A55) is used to relate the density functions and the distribution functions.

\section{THE $z$-INDEPENDENT APPROXIMATION}

Consider a long, cylindrical, non-neutral plasma for which we assume no dependence on the coordinate $z$ along the longitudinal direction. Specifically, we assume a perturbation to an equilibrium potential of the form $\Phi=\Phi^{(0)}(r)$ $+\Phi^{(1)}(r, \phi, t)$, with corresponding perturbations in the density $n(\mathbf{x}, t)=n^{(0)}(r)+n^{(1)}(r, \phi, t)$. Because of the linearity in the Vlasov equation and Poisson's equation, we may consider the axial modes one at a time, recognizing that the full perturbation will be a sum over such modes. We assume an exponential dependence on time and write this perturbation in the form $\Phi^{(1)}=\phi^{(1)}(r) \exp (-i \omega t+i m \varphi)$. From the definition of $\mathbf{v}_{e}$ we find

$$
\begin{aligned}
& \mathbf{v}_{e}^{(0)}=\frac{q}{M \Omega} \frac{d \Phi^{(0)}}{d r} \hat{\varphi} \equiv r \omega_{0}(r) \hat{\varphi} \\
& \mathbf{v}_{e}^{(1)}=\frac{q}{M \Omega}\left[\frac{-i m}{r} \phi^{(1)} \hat{\mathbf{r}}+\phi^{(1) \prime} \hat{\varphi}\right] e^{-i \omega t+i m \varphi} .
\end{aligned}
$$

With $\mathbf{v}_{e}^{(0)}$ and $\mathbf{v}_{e}^{(1)}$ as given in Eqs. (15) and (16) (but in GC coordinates) it is straightforward to find from Eq. (10) that $\dot{U}^{(0)}=\dot{U}^{(1)}=0$ and from Eq. (7) that

$$
\begin{aligned}
\dot{\mathbf{X}}^{(0)}= & U \hat{\mathbf{Z}}+\hat{\Phi}\left[R \omega_{0}\left(1-\epsilon \frac{\omega_{0}}{\Omega}-\epsilon^{2} \frac{\omega_{0} \omega_{p}^{2}}{\Omega^{3}}\right)-\epsilon^{2} \frac{W^{2} \omega_{p}^{2} h^{\prime}}{4 \Omega^{3}}\right]+\mathcal{O}\left(\epsilon^{3}\right), \\
\dot{\mathbf{X}}^{(1)}= & \left(\frac{q}{M \Omega}\right) e^{-i \omega t+i m \Phi}\left\{i \hat { \mathbf { R } } \left[-\frac{m}{R} \phi^{(1)}+\frac{\epsilon}{\Omega}\left\{\phi^{(1) \prime}\left(\omega-m \omega_{0}\right)-\frac{m}{R} \phi^{(1)} \frac{\omega_{p}^{2}}{\Omega}\right\}+\frac{\epsilon^{2}}{\Omega^{2}}\left\{-\frac{W^{2} m}{4 R} D^{2} \phi^{(1)}+\frac{\omega_{p}^{2}}{\Omega}\left[\phi^{(1) \prime}(\omega\right.\right.\right.\right. \\
& \left.\left.\left.\left.-m \omega_{0}\right)-\frac{m \omega_{p}^{2}}{R \Omega} \phi^{(1)}\right]\right\}\right]+\hat{\Phi}\left[\phi^{(1) \prime}-\frac{\epsilon}{\Omega}\left\{2 \omega_{0} \phi^{(1) \prime}+\frac{m}{R} \phi^{(1)}\left(\omega-m \omega_{0}\right)\right\}+\frac{\epsilon^{2}}{\Omega^{2}}\left\{\frac{W^{2}}{4}\left[D^{2} \phi^{(1)}\right]^{\prime}+R \omega_{0}^{2} D^{2} \phi^{(1)}\right.\right. \\
& \left.\left.\left.-\frac{\omega_{p}^{2}}{\Omega}\left(2 \omega_{0} \phi^{(1) \prime}+\frac{m}{R} \phi^{(1)}\left(\omega-m \omega_{0}\right)\right)\right\}\right]\right\}+\mathscr{Q}\left(\epsilon^{3}\right) .
\end{aligned}
$$

In Eqs. (17) we have used $h^{\prime}=d \ln n^{(0)} / d R$, the usual definition for the plasma frequency profile $\omega_{p}^{2}=4 \pi q^{2} n^{(0)} / M$ and Poisson's equation for the equilibrium field in the form

$$
\frac{1}{r} \frac{d}{d r}\left(r^{2} \omega_{0}\right)=-\frac{\omega_{p}^{2}}{\Omega}
$$

Also for brevity in Eq. (18) we have defined

$$
D^{2} \phi^{(1)} \equiv \frac{1}{R} \frac{d}{d R}\left(R \frac{d \phi^{(1)}}{d R}\right)-\frac{m^{2}}{R^{2}} \phi^{(1)}
$$

Since $U^{(0)}$ is constant, the Vlasov equation for $F^{(0)}$ is satisfied by a Maxwellian distribution function of the form $F^{(0)}=f(R) \exp \left(-\left(W^{2}+U^{2}\right) / v_{T}^{2}\right) /\left(M v_{T} \sqrt{\pi}\right)^{3}$, where $v_{T}$ is the thermal velocity given by $v_{T}=\sqrt{(2 k T / M)}$.

The GC distribution functions are used to obtain the particle densities using the result from the Lie transformation analysis of the appendix:

$$
\begin{aligned}
n(\mathbf{x}, t)= & \mathscr{T}_{1}(F)+\epsilon \frac{(\hat{\mathbf{b}} \cdot \boldsymbol{\psi})}{\Omega}\left\{\mathscr{T}_{1}(F)-\frac{1}{2} \mathscr{T}_{2}(F, W)\right\}+\frac{\epsilon^{2}}{\Omega^{2}}\left\{\frac{1}{4} \mathscr{T}_{0}\left(\left[\frac{D \mathbf{v}_{e}}{D t}+v_{\|} \boldsymbol{\psi}_{\perp}\right]^{2} F, W\right)-\mathscr{T}_{1}\left(\left[\frac{D \mathbf{v}_{e}}{D t}+v_{\|} \boldsymbol{\psi}_{\perp}\right] \cdot \nabla F\right)\right. \\
& +\frac{1}{4} \mathscr{T}_{1}\left(\left[\frac{D \mathbf{v}_{e}}{D t}+v_{\|} \boldsymbol{\psi}_{\perp}\right]^{2} F, W W\right)+\mathscr{T}_{2}(F, W)\left[-\frac{5}{64}(\hat{\mathbf{b}} \cdot \boldsymbol{\psi})^{2}-\frac{13}{32} \nabla \cdot\left(\mathbf{v}_{e} \cdot \nabla \mathbf{v}_{e}\right)-\frac{1}{2} \boldsymbol{\psi}_{\perp} \cdot \nabla v_{\|}+\frac{1}{2} v_{\|}(b \psi b)-\frac{1}{2} \psi_{\perp}^{2}\right] \\
& -\mathscr{T}_{2}\left(\left[\frac{D \mathbf{v}_{e}}{D t}+v_{\|} \boldsymbol{\psi}_{\perp}\right] \cdot \nabla F, W\right)+\frac{1}{2}(\hat{\mathbf{b}} \times \boldsymbol{\psi}) \cdot \mathscr{T}_{2}\left(\frac{D \mathbf{v}_{e}}{D t} F,_{U W}\right)+\mathscr{T}_{3}(F, W W)\left[\frac{9}{64}(\hat{\mathbf{b}} \cdot \boldsymbol{\psi})^{2}+\frac{1}{32} \nabla \cdot\left(\mathbf{v}_{e} \cdot \nabla \mathbf{v}_{e}\right)\right]+\nabla_{\perp}^{2} \mathscr{T}_{3}(F) \\
& \left.+(\boldsymbol{\psi} \times \hat{\mathbf{b}}) \cdot \nabla \mathscr{T}_{3}\left(F,,_{U}\right)+\frac{1}{4} \psi_{\perp}^{2} \mathscr{T}_{3}\left(F,,_{U U}\right)\right\},
\end{aligned}
$$


where for brevity the velocity integrals are written in the form

$$
\mathscr{T}_{k}(\cdots) \equiv 2 \pi M^{3} \int_{-\infty}^{+\infty} d U \int_{0}^{\infty}(\ldots) W^{k} d W .
$$

It can be desirable to relate $f(r)$ to a known equilibrium particle density $n^{(0)}(r)$. This can be done by writing $f(r)$ in the form $f(r)=f_{0}(r)+\epsilon f_{1}(r)+\epsilon^{2} f_{2}(r)$ and substituting this form into the equation for $n^{(0)}$ obtained from Eq. (21). Performing the integrations over the phase space coordinates $(U, W)$, determines order by order $f_{0}(r), f_{1}(r)$, and $f_{2}(r)$ in terms of the particle density profile $n^{(0)}$. It is straightforward to find

$$
\begin{aligned}
f_{0}(r)= & n^{(0)}(r) \\
f_{1}(r)= & 2 \frac{\omega_{p}^{2}}{\Omega^{2}} n^{(0)} \\
f_{2}(r)= & {\left[3 \frac{\omega_{p}^{4}}{\Omega^{4}}-2 \frac{\omega_{0}^{2}}{\Omega^{2}}-2 \frac{\omega_{0} \omega_{p}^{2}}{\Omega^{3}}\right] n^{(0)}+\frac{r \omega_{0}^{2}}{\Omega^{2}} \frac{d n^{(0)}}{d r} } \\
& -\rho^{2} \frac{1}{r} \frac{d}{d r}\left(r \frac{d n^{(0)}}{d r}\right),
\end{aligned}
$$

where we denote for the gyroradius squared $\rho^{2}=v_{T}^{2} / \Omega^{2}$.

To find the perturbed distribution function $F^{(1)}$ we regard the left-hand side of Eq. (14) as the total time derivative of the perturbed distribution function over the unperturbed orbits in the usual way (see Ref. 12). From Eq. (17) we find that the unperturbed orbits are characterized to $\mathscr{Q}\left(\epsilon^{3}\right)$ at time $t^{\prime}<t$ by

$$
\begin{aligned}
& R=\text { const } \\
& \Phi\left(t^{\prime}\right)=\Phi-\bar{\omega}(R)\left(t-t^{\prime}\right), \\
& Z\left(t^{\prime}\right)=Z-U\left(t-t^{\prime}\right),
\end{aligned}
$$

where

$$
\bar{\omega}(R, W)=g \omega_{0}-\epsilon^{2} \frac{W^{2} \omega_{p}^{2} h^{\prime}}{4 R \Omega^{3}}+\odot\left(\epsilon^{3}\right),
$$

with

$$
g=1-\epsilon \frac{\omega_{0}}{\Omega}-\epsilon^{2} \frac{\omega_{0} \omega_{p}^{2}}{\Omega^{3}} .
$$

Carrying out the integration over the unperturbed orbits in Eq. (14) gives the perturbed distribution function. Written in laboratory coordinates as needed for use in Eq. (21),

$$
\begin{aligned}
F^{(1)}(r, \varphi, t)= & \left(\frac{q}{M \Omega}\right) \frac{\exp (-i \omega t+i m \varphi)}{(\omega-m \bar{\omega})} \frac{d F^{(0)}}{d r}\left\{-\frac{m}{r} \phi^{(1)}+\frac{\epsilon}{\Omega}\left[\phi^{(1) \prime}(\omega-m \bar{\omega})-\frac{\omega_{0}^{2}}{\Omega} \phi^{(1) \prime}-\frac{m \omega_{p}^{2}}{r \Omega} \phi^{(1)}\right]+\frac{\epsilon^{2}}{\Omega^{2}}\right. \\
& \left.\times\left[-\frac{m W^{2}}{4 r} D^{2} \phi^{(1)}+\frac{\omega_{p}^{2}}{\Omega}\left(\phi^{(1) \prime}(\omega-m \bar{\omega})-\frac{m \omega_{p}^{2}}{r \Omega} \phi^{(1)}\right)\right]\right\}+\mathscr{O}\left(\epsilon^{3}\right) .
\end{aligned}
$$

With this perturbed distribution function in hand we can substitute into Eq. (21) to find the perturbed density,

$$
\begin{aligned}
n^{(1)}(\mathbf{x}, t)= & \frac{q n^{(0)}}{M \Omega^{2}} e^{-i \omega t+i m \varphi}\left\{-\frac{m}{r} \frac{f^{\prime}}{n^{(0)}} \phi^{(1)} I_{1}+\epsilon\left[-\frac{m}{r} \tilde{\omega}_{p}^{2} \frac{f^{\prime}}{n^{(0)}} \phi^{(1)} I_{1}+\frac{f^{\prime}}{n^{(0)}} \phi^{(1) \prime}\left(1-\tilde{\omega}_{0}^{2} I_{1}\right)+2 \frac{f}{n^{(0)}} D^{2} \phi^{(1)}\right]+\epsilon^{2}\right. \\
& \times\left[-\frac{m}{r} \phi^{(1)}\left(\frac{2 m}{r} \frac{f}{n^{(0)}}\left(\tilde{\omega}_{0}+\tilde{\omega}_{p}^{2}\right)+\frac{f^{\prime}}{n^{(0)}}\left(\tilde{\omega}-m g \tilde{\omega}_{0}\right)-\frac{m^{2} \rho^{2}}{r^{2}} I_{2}+2 I_{1} \tilde{\omega}_{0}\left(\tilde{\omega}_{0}+\tilde{\omega}_{p}^{2}\right)\right)-\phi^{(1)} \frac{f^{\prime}}{n^{(0)}}\left(\tilde{\omega}_{p}^{2}+2 \tilde{\omega}_{0}\right.\right. \\
& \left.\left.\left.-2 \tilde{\omega}_{0}^{2} \tilde{\omega}_{p}^{2} I_{1}\right)+\frac{r m \tilde{\omega}_{0}^{2}}{n^{(0)}} \frac{d}{d r}\left(\frac{f^{\prime}}{r} \phi^{(1)} I_{1}\right)-D^{2} \phi^{(1)}\left(\frac{f}{n^{(0)}} \tilde{\omega}_{p}^{2}+\frac{m}{4 r} \rho^{2} \frac{f^{\prime}}{n^{(0)}} I_{2}\right)-\frac{m \rho^{2}}{r n^{(0)}} \frac{d}{d r}\left(r \frac{d}{d r}\left(\frac{f^{\prime}}{r} \phi^{(1)} I_{2}\right)\right)\right]\right\},
\end{aligned}
$$

where a tilde over an angular frequency denotes that it has been scaled by the cyclotron frequency $\Omega$. The phase space integrals $I_{1}$ and $I_{2}$ are defined as

$$
\begin{aligned}
& \frac{v_{T}^{2}}{2 \Omega} I_{1}=\int_{0}^{\infty} \frac{W \exp \left(-W^{2} / v_{T}^{2}\right)}{\omega-m \bar{\omega}(r, W)} d W, \\
& \frac{v_{T}^{4}}{2 \Omega} I_{2}=\int_{0}^{\infty} \frac{W^{3} \exp \left(-W^{2} / v_{T}^{2}\right)}{\omega-m \bar{\omega}(r, W)} d W .
\end{aligned}
$$

We now substitute $n^{(1)}(\mathbf{x}, t)$ from Eq. (28) into Poisson's equation to obtain the mode equation for the potential perturbation $\phi^{(1)}$ for the $m$ axial mode,

$$
\begin{gathered}
b_{0}\left[\frac{1}{r} \frac{d}{d r}\left(r \frac{d \phi^{(1)}}{d r}\right)-\frac{m^{2}}{r^{2}} \phi^{(1)}\right]-\frac{b_{1}}{r^{2}} \phi^{(1)}-\frac{b_{2}}{r} \frac{d \phi^{(1)}}{d r} \\
-b_{3} r \frac{d}{d r}\left[r \frac{d}{d r}\left(\frac{1}{r} \phi^{(1)} f^{\prime} I_{2}\right)\right] \\
-b_{4} r \frac{d}{d r}\left(\frac{1}{r} \phi^{(1)} f^{\prime} I_{1}\right)=0,
\end{gathered}
$$

where the coefficients are

$$
b_{0}=1+2 \epsilon \frac{f}{n^{(0)}} \tilde{\omega}_{p}^{2}-\epsilon^{2}\left(\frac{f}{n^{(0)}} \tilde{\omega}_{p}^{4}+\frac{m}{4 r} \tilde{\omega}_{p}^{2} \rho^{2} \frac{f^{\prime}}{n^{(0)}} I_{2}\right),
$$




$$
\begin{aligned}
b_{1}= & r m \tilde{\omega}_{p}^{2}\left\{\frac{f^{\prime}}{n^{(0)}} I_{1}\left[1-\epsilon \tilde{\omega}_{p}^{2}+2 \epsilon^{2} \tilde{\omega}_{0}\left(\tilde{\omega}_{0}+\tilde{\omega}_{p}^{2}\right)\right]\right. \\
& +2 \epsilon^{2} \frac{m f}{r n^{(0)}}\left(\tilde{\omega}_{0}+\tilde{\omega}_{p}^{2}\right) \\
& \left.+\epsilon^{2} \frac{f^{\prime}}{n^{(0)}}\left(\tilde{\omega}-m g \tilde{\omega}_{0}-m^{2} \frac{\rho^{2}}{r^{2}} I_{2}\right)\right\}, \\
b_{2}= & -\frac{r f^{\prime}}{n^{(0)}} \tilde{\omega}_{p}^{2}\left[\epsilon\left(1-\tilde{\omega}_{0}^{2} I_{1}\right)-\epsilon^{2}\left(\tilde{\omega}_{p}^{2}+2 \tilde{\omega}_{0}\right.\right. \\
& \left.\left.-2 \tilde{\omega}_{p}^{2} \tilde{\omega}_{0}^{2} I_{1}\right)\right], \\
b_{3}= & \frac{\epsilon^{2} m \tilde{\omega}_{p}^{2} \rho^{2}}{r^{2} n^{(0)}}, \\
b_{4}= & -\frac{\epsilon^{2} m \tilde{\omega}_{p}^{2} \tilde{\omega}_{0}^{2}}{n^{(0)}} .
\end{aligned}
$$

We note that the last two terms in Eq. (31) could be expanded and incorporated into the other terms, giving modified forms for $b_{0}, b_{1}$, and $b_{2}$. Equation (31) is an eigenvalue equation for each $m$, second order in the derivatives of the perturbed potential function $\phi^{(1)}$ and is a convenient form for a subsequent numerical study.

Comparing the mode equation, Eq. (31), and the coefficient functions with the familiar equation from a cold-fluid model [see for example Eq. (11) of Ref. 13], we see that resonance occurs with a Doppler shifted rotation frequency, $g \tilde{\omega}_{0}=\left(1-\epsilon \tilde{\omega}_{0}-\epsilon^{2} \tilde{\omega}_{0} \tilde{\omega}_{p}^{2}\right) \tilde{\omega}_{0} . \quad$ In the limit $\epsilon \rightarrow 0$, $I_{1} \rightarrow 1 /\left(\tilde{\omega}-m \tilde{\omega}_{0}\right)$ and becomes a simple pole consistent with the cold-fluid approximation. In order to elucidate the singular nature of the phase space integrals $I_{1}$ and $I_{2}$, we look closer at their properties. For brevity we define $G=\epsilon^{2} m \rho^{2} \tilde{\omega}_{p}^{2} h^{\prime} / 4 r$ and then let

$$
z=\left(\tilde{\omega}-m g \tilde{\omega}_{0}\right) / G \text {. }
$$

The quantity $z$ can, of course, be complex if the eigenvalue $\omega$ has an imaginary part indicating mode damping or growth. Letting $\xi=W^{2} / v_{T}^{2}$, then $I_{1}=I / G$ where

$$
I=\int_{0}^{\infty} \frac{e^{-\xi} d \xi}{z+\xi} .
$$

Note also that

$$
I_{2}=\frac{1}{G} \int_{0}^{\infty} \frac{\xi e^{-\xi}}{z+\xi} d \xi=\frac{1}{G}(1-z I) .
$$

In the notation of Abramowitz and Stegun, Ref. 14, the integral $I$ is given by

$$
I=e^{z} E_{1}(z),
$$

with

$$
E_{1}(z)=-\gamma-\ln z-\sum_{n=1}^{\infty} \frac{(-1)^{n} z^{n}}{n n !}, \quad|\arg z|<\pi,
$$

where $\gamma=0.57721 \cdots$ is Euler's constant. ${ }^{14}$ Asymptotically $I \sim 1 / z$ and thus even though $I_{1}$ as it occurs in Eq. (31) asymptotically behaves like a pole, as in the cold-fluid approximation, ${ }^{13}$ the singularity has been changed by the in- clusion of FLR effects from a simple pole to a logarithmic singularity. Furthermore, when $z<0$ the integral $I_{1}$ will necessarily have an imaginary piece, and this must happen when the profile is hollow and $n^{(0)}$ has a vanishing derivative at some point in the interval. This then forces $\omega$ to have a nonvanishing imaginary part and the perturbed potential $\phi^{(1)}$ is complex. This change from a pole to the integral $I_{1}$ is the most significant qualitative change in the mode equation from the cold-fluid form and in a subsequent paper we hope to explore in detail the full implications of this change for the spectrum of Eq. (31).

\section{NUMERICAL INVESTIGATIONS OF THE $m=1$ MODE}

To obtain a numerical solution to the mode equation (31), we expand $\phi^{(1)}(r)$ as a sum of cubic B-splines, $\phi^{(1)}(r)=\sum c_{i} \psi_{i}(r)$, for a finite element partition of the interval $\left[0, r_{\text {wall }}\right]$. Then applying a standard Galerkin technique to Eq. (31) and normalizing the eigenfunction $\phi^{(1)}(r)$ at some intermediate point in the interval leads to a standard eigenvalue problem for the eigenvalue $\omega$ and the eigenvector of expansion coefficients $c_{i}$. As is evident from the dependencies in Eq. (31) and the equations for the coefficient functions that follow, the eigenfrequencies and functions depend on the temperature, the magnetic field, and the equilibrium profile $n^{(0)}(r)$. We choose values corresponding to those reported in Ref. 1 and a similar profile $n^{(0)}(r)$. Specifically we choose a temperature of $T=1.2 \mathrm{keV}$, a magnetic field of $B=375.0 \mathrm{G}$, and a density profile given by the function

$$
n^{(0)}(r)=\hat{n}\left[1+\mu_{1}\left(r / r_{p}\right)^{2}\right] \exp \left[-\left(r / r_{p}\right)^{\mu_{2}}\right]
$$

where $\hat{n}$ is the central density, $\mu_{1}$ controls the hollowness of the profile, $\mu_{2}$ controls the steepness of the cutoff, and $r_{p}$ controls the position of the cutoff. We used the values $\hat{n}=3.6 \times 10^{6} \mathrm{~cm}^{-3}, \mu_{1}=0.95, \mu_{2}=8, r_{\text {wall }}=3.81 \mathrm{~cm}$, and $r_{p} / r_{\text {wall }}=0.47$, resulting in a profile that is a reasonable match to that reported by Driscoll. ${ }^{1}$ Denoting $\omega_{\max }=1.098 \times 10^{6} \mathrm{~s}^{-1}$ as the maximum value of $g \omega_{0}$, our investigation of modes for Eq. (31) was undertaken primarily in the neighborhood of $\omega_{\max }$, except for verifying the existence of the neutrally stable diocotron (wall) mode. In the neighborhood of $\omega_{\max }, \operatorname{Re}(\omega) \sim \omega_{\max }$, we find only modes with growth rates $\operatorname{Im}(\omega) \sim 1-2 \times 10^{-3} \times \omega_{\max }$, which are about a factor of 10 lower than those seen in Driscoll's experiment. As one check on the code performance we kept only terms corresponding to the model considered by $\mathrm{Smith}^{3}$ and successfully duplicated his results. The growth rates found by fully including all FLR corrections in Eq. (31) fall between those found by $\mathrm{Smith}^{3}$ and those reported in Ref. 4 . We conclude from this study that FLR, i.e., finite temperature effects, have little to do with the exponentially growing instability observed by Driscoll. ${ }^{1}$ 
In passing we note that setting all terms of $\mathscr{Q}\left(\epsilon^{2}\right)$ equal to zero, but keeping terms of $\mathscr{Q}(\epsilon)$ corresponds to keeping only corrections corresponding to the polarization drift and returning the singular integral $I_{1}$ to a pole. For the magnetic field value chosen above and the indicated profile, the marginally stable, cold-fluid mode with $\operatorname{Re}(\omega)=\omega_{\max }$ becomes unstable with a growth rate roughly half that obtained with the full FLR effects included. Leaving everything else the same but replacing the pole with the singular integral $I_{1}$, reduces the growth again by about $40 \%$. Qualitatively, the most important change introduced by including FLR effects is the change from the pole singularity, $1 /\left(\tilde{\omega}-m g \tilde{\omega}_{0}\right)$ to the logarithmic singularity of the integral $I_{1}$. This implies that it is no longer possible to have $\operatorname{Im}\left(\phi^{(1)}(r)\right)=0$.

The correction terms in Eq. (31) that are most important for giving the computed growth are those in the coefficient functions $b_{0}$ and $b_{3}$. The terms with coefficients $b_{2}$ and $b_{4}$ are unimportant and the corrections terms in $b_{1}$ make a difference on the order of $10 \%$.

\section{ACKNOWLEDGMENT}

The author acknowledges enlightening discussions with my colleague Ross Spencer on many aspects of this work, particularly in regards to the behavior of the phase space integrals.

\section{APPENDIX: LIE TRANSFORMATION TO GC COORDINATES}

The fundamental ideas behind the usage of a Lagrangian in extended phase space have been thoroughly discussed in Refs. 5, 6, and 10. Following this procedure, we view the Lagrangian in Eq. (1) as a 1-form on extended phase space. Furthermore, we view this Lagrangian as an expansion in the ordering parameter $\epsilon$, where $\omega / \Omega, \rho / L$ are $\mathscr{Q}(\epsilon)$. We write

$$
\gamma=\frac{1}{\epsilon} \sum_{n=0}^{\infty} \epsilon^{n} \gamma^{(n)},
$$

where from Eq. (1) we identify

$$
\begin{aligned}
& \gamma_{i}^{(0)}=\frac{M \Omega}{2} \epsilon_{i j k} b^{j} x^{k}, \\
& \gamma_{u}^{(0)}=\gamma_{\theta}^{(0)}=\gamma_{w}^{(0)}=0, \gamma_{t}^{(0)}=-q \Phi(\mathbf{x}, t), \\
& \gamma_{i}^{(1)}=M u b_{i}+m w c_{i}+M v_{E i}, \\
& \gamma_{u}^{(1)}=\gamma_{\theta}^{(1)}=\gamma_{w}^{(1)}=0, \\
& \gamma_{t}^{(1)}=-\frac{1}{2}\left(u^{2}+w^{2}+v_{e}^{2}\right)-M w \hat{\mathbf{c}} \cdot \mathbf{v}_{e} .
\end{aligned}
$$

All other $\gamma^{(i)}$ are zero. We recall that the phase space coordinates are $(\mathbf{x}, u, \theta, w, t)$ and other notations as defined in Section I. The Latin vector indices $i, j, k, l, m, \ldots$, run over $1,2,3$ and refer only to spatial coordinates. Frequently the spatial part of phase space vectors will be denoted with bold type, e.g. $g$ denotes the spatial part of the phase space vector field $g$.
We represent the Lie derivative operator with respect to the vector field $g$ in the form $L_{g}$. The succession of Lie transformations corresponding to increasingly higher order is given by

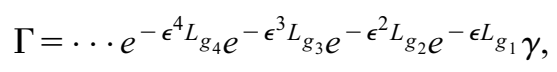

where the transformed 1-form (Lagrangian) is also ordered in $\epsilon$, i.e., $\Gamma=\frac{1}{\epsilon} \sum_{n=0}^{\infty} \epsilon^{n} \Gamma^{(n)}$. Representing the exponential operators in Eq. (A5) as a power series in the Lie derivatives and matching order by order gives

$$
\begin{aligned}
& \Gamma^{(0)}=\gamma^{(0)}+\mathbf{d} F^{(0)}, \\
& \Gamma^{(1)}=\gamma^{(1)}-L_{g_{1}} \gamma^{(0)}+\mathbf{d} F^{(1)}, \\
& \Gamma^{(2)}=\gamma^{(2)}-L_{g_{1}} \gamma^{(1)}+L_{2} \gamma^{(0)}+\mathbf{d} F^{(2)}, \\
& \Gamma^{(3)}=\gamma^{(3)}-L_{g_{1}} \gamma^{(2)}+L_{2} \gamma^{(1)}+L_{3} \gamma^{(0)}+\mathbf{d} F^{(3)}, \\
& \Gamma^{(4)}=\gamma^{(4)}-L_{g_{1}} \gamma^{(3)}+L_{2} \gamma^{(2)}+L_{3} \gamma^{(1)}+L_{4} \gamma^{(0)}+\mathbf{d} F^{(4)},
\end{aligned}
$$

where

$$
\begin{aligned}
& L_{2}=\frac{1}{2}\left(L_{g_{1}}\right)^{2}-L_{g_{2}}, \\
& L_{3}=L_{g_{2}} L_{g_{1}}-\frac{1}{6}\left(L_{g_{1}}\right)^{3}-L_{g_{3}}, \\
& L_{4}=\frac{1}{24}\left(L_{g_{1}}\right)^{4}+\frac{1}{2}\left(L_{g_{2}}\right)^{2}-\frac{1}{2} L_{g_{2}}\left(L_{g_{1}}\right)^{2}+L_{g_{3}} L_{g_{1}}-L_{g_{4}} .
\end{aligned}
$$

We denote components in phase space with Greek letters $\mu, \nu, \ldots$, and repeated indices denote a sum in the usual way. For purposes of brevity in computation it is convenient to write

$$
\left[L_{g_{i}} \gamma^{(k)}\right]_{\mu}=g_{i}^{\nu}\left(\gamma_{\mu, \nu}^{(k)}-\gamma_{\nu, \mu}^{(k)}\right) \equiv g_{i}^{\nu} \omega_{\nu \mu}^{(k)} .
$$

In this equation $i$ labels which generator is used in the Lie derivative and the index $k$ labels which 1-form in the $\epsilon$-expansion. The indices $\mu$ and $\nu$ are phase space coordinate labels. The comma denotes partial differentiation, i.e., $\gamma_{\mu, \nu}^{(k)}=\partial \gamma^{(k)} / \partial x^{\nu}$ with $x^{\nu}$ one of the phase space coordinates. In a similar fashion we define

$$
\begin{aligned}
{\left[\left(L_{g_{i}}\right)^{2} \gamma^{(k)}\right]_{\mu} } & =g_{i}^{\nu}\left(g_{i, \nu}^{\sigma} \omega_{\sigma \mu}^{(k)}-g_{i, \mu}^{\sigma} \omega_{\sigma \nu}^{(k)}+g_{i}^{\sigma} \omega_{\nu \mu, \sigma}^{(k)}\right) \\
& \equiv g_{i}^{\nu} \bar{\omega}_{\nu \mu}^{(k, i)}, \\
{\left[\left(L_{g_{i}}\right)^{3} \gamma^{(k)}\right]_{\mu} } & \equiv g_{i}^{\nu} \overline{\bar{\omega}}_{\nu \mu}^{(k, i)},
\end{aligned}
$$

where $\overline{\bar{\omega}}_{\nu \mu}^{(k, i)}$ is obtained from the definition of $\bar{\omega}_{\nu \mu}^{(k, i)}$ given in Eq. (A9) by replacing $\omega^{(k)}$ with $\bar{\omega}^{(k, i)}$ in that definition,

$$
\left[\left(L_{g_{i}}\right)^{4} \gamma^{(k)}\right]_{\mu} \equiv g_{i}^{\nu} \overline{\bar{\omega}}_{\nu \mu}^{(k, i)},
$$

where $\overline{\overline{\bar{\omega}}}^{(k, i)}$ is obtained from $\overline{\bar{\omega}}^{(k, i)}$ just as $\overline{\bar{\omega}}^{(k, i)}$ is obtained from $\bar{\omega}^{(i, i)}$; 


$$
\left[L_{g_{i}} L_{g_{j}} \gamma^{(k)}\right]_{\mu}=g_{i}^{\nu} \tilde{\omega}_{\nu \mu}^{(k, j)},
$$

where

$$
\tilde{\omega}_{\nu \mu}^{(k, j)} \equiv g_{j, \nu}^{\sigma} \omega_{\sigma \mu}^{(k)}-g_{j, \mu}^{\sigma} \omega_{\sigma \nu}^{(k)}+g_{j}^{\sigma}\left(\omega_{\sigma \mu, \nu}^{(k)}-\omega_{\sigma \nu, \mu}^{(k)}\right) .
$$

Likewise

$$
\left[L_{g_{i}}\left(L_{g_{j}}\right)^{2} \gamma^{(k)}\right]_{\mu}=g_{j}^{\nu} \tilde{\omega}_{\nu \mu}^{(k, i)},
$$

where $\tilde{\omega}^{(k, i)}$ is obtained from $\bar{\omega}^{(k, i)}$ as $\tilde{\omega}^{(k, i)}$ is from $\omega^{(k)}$. The calculation of the transformed 1-forms, $\Gamma^{(i)}$, given in Eqs. (A6) and (A7) is simplified considerably by using the antisymmetric tensors given in Eqs. (A9)-(A14).

The hierarchy of Eqs. (A6) and (A7) is used in the following way: the vector fields $g_{1}, g_{2}, \ldots$, and the gauge functions $F^{(0)}, F^{(1)}, \ldots$, are chosen level by level to eliminate the dependence of the Lagrangian 1-forms on the gyroangle $\theta$. This objective does not fully specify the functional dependence of the generating vector fields or the gauge functions, but nearly so. Simplicity arguments complete the specification. Despite our intent to only obtain the GC transformation to $\mathscr{Q}\left(\epsilon^{2}\right)$ so that FLR effects may be properly included, it soon becomes apparent that to fully determine the GC transformation to $\mathscr{O}\left(\epsilon^{2}\right)$ one must carry the transformation of the Lagrangian 1-forms to $\mathcal{Q}\left(\epsilon^{4}\right)$.

Once the generating vector fields $g_{1}, g_{2}, \ldots$ have been determined, then the corresponding coordinate transformation in phase space is also determined. Actually, it is the inverse of this coordinate transformation that is needed. Denote the new (phase space) GC coordinates as $Z^{\mu}$ and the old phase space coordinates as $z^{\mu}$,

$$
z^{\mu}=\left(e^{-\epsilon L_{g_{1}}} e^{-\epsilon^{2} L_{g_{2}} \ldots}\right) Z^{\mu} .
$$

Using the fact that $L_{g_{i}}\left(Z^{\mu}\right)=g_{i}^{\mu}$, the power series expansions of the exponential operators, and keeping only to $\mathscr{Q}\left(\epsilon^{2}\right)$ gives

$$
z^{\mu}=Z^{\mu}-\epsilon g_{1}^{\mu}+\epsilon^{2}\left(\frac{1}{2} g_{1}^{\nu} \frac{\partial g_{1}^{\mu}}{\partial Z^{\nu}}-g_{2}^{\mu}\right)+\mathscr{Q}\left(\epsilon^{3}\right) .
$$

Once the gyrophase has been eliminated to the required order through Lie transformations and this transformation is known, the problem of solving the Vlasov equation in GC coordinates can be addressed. In covariant form the Vlasov equation is

$$
\frac{d F}{d \lambda}+\frac{d Z^{\mu}}{d \lambda} \frac{\partial F}{\partial Z^{\mu}}=0
$$

where $\lambda$ is just some convenient parameter marking the path of a particle in extended phase space. In the familiar GC coordinates and with time $t$ chosen as the path parameter this simplifies to Eq. (12).

Knowing the distribution function $F(\mathbf{X}, U, W, t)$ then allows us to obtain the particle density distribution in space through the result

$$
\begin{aligned}
n(\mathbf{x}, t)= & M^{3} \int T F(\mathbf{X}, U, \Theta, W, t) \delta^{3}(\mathbf{x} \\
& \left.-\mathbf{x}^{\prime}(\mathbf{X}, U, \Theta, W)\right) \frac{\partial(\mathbf{x}, u, \theta, w)}{\partial(\mathbf{X}, U, \Theta, W)} d^{6} Z,
\end{aligned}
$$

where $d^{6} Z=W d W d U d \Theta d^{3} X, \partial(\ldots) / \partial(\ldots)$ denotes the Jacobian of the transformation, and $\operatorname{TF}(\mathbf{X}, U \Theta, W, t)$ denotes the transformed distribution function having exactly the same value at the $\mathrm{GC}$ coordinates $Z^{\mu}$ as the distribution function $f\left(\mathbf{x}^{\prime}, u, \theta, w, t\right)$ has at $z^{\mu}$,

$$
T F=\cdots e^{\epsilon^{2} L_{g_{2}}} e^{\epsilon L_{1} F} .
$$

Writing the exponentials as a power series and keeping only to $\mathcal{Q}\left(\epsilon^{2}\right)$, Eq. (19) gives

$$
\begin{aligned}
T F= & F+\epsilon g_{1}^{\mu} \frac{\partial F}{\partial Z^{\mu}}+\epsilon^{2}\left[g_{2}^{\mu} \frac{\partial F}{\partial Z^{\mu}}+\frac{1}{2} g_{1}^{\nu} \frac{\partial}{\partial Z^{\nu}}\left(g_{1}^{\mu} \frac{\partial F}{\partial Z^{\mu}}\right)\right] \\
& +\mathcal{O}\left(\epsilon^{3}\right) .
\end{aligned}
$$

With this sketch in place as to the manner in which the calculation proceeds we now supply some details. We omit lengthy algebra, giving only results when the procedure is clear. First we focus on the details of obtaining the GC transformation to $\mathscr{Q}\left(\epsilon^{2}\right)$. For brevity we use the convenient notation for arbitrary vectors $\mathbf{A}, \mathbf{B}, \mathbf{C}$,

$$
(A B C) \equiv \mathbf{A} \cdot \nabla \mathbf{B} \cdot \mathbf{C}=A_{i} B_{j, i} C_{j} .
$$

Also for convenience we define

$$
\boldsymbol{\psi}=\nabla \times \mathbf{v}_{e}, \quad v_{\|}=\frac{q}{M \Omega}(\hat{\mathbf{b}} \cdot \mathbf{E}) .
$$

Before beginning the procedure for solving for the various $g$ 's we make a preparatory transformation using the vector field $g_{p}$ where

$$
g_{p}^{i}=-\frac{w}{\Omega} a^{i},
$$

and all other phase space components of $g_{p}$ are zero. We compute the various Lie derivatives needed for Eqs. (A6) as defined in Eqs. (A7)-(A14). The gauge functions in Eqs. (A6) are, of course, zero for the preparatory transformation, which is a specified transformation, not one in the process of being determined.

Using the preparatory transformation, the Lie transformed Lagrangian 1-forms become

$$
\begin{aligned}
& \overline{\Gamma_{i}^{(0)}}=\frac{m \Omega}{2} \epsilon_{i j k} b^{j} x^{k}, \\
& \bar{\Gamma}_{u}^{(0)}=\overline{\Gamma_{\theta}^{(0)}}=\overline{\Gamma_{w}^{(0)}}=0, \overline{\Gamma_{t}^{(0)}}=-q \Phi(\mathbf{x}, t), \\
& \bar{\Gamma}_{i}^{(1)}=M u b_{i}+M v_{E i}, \\
& \bar{\Gamma}_{u}^{(1)}=\overline{\Gamma_{\theta}^{(1)}}=\overline{\Gamma_{w}^{(1)}}=0, \\
& {\overline{\Gamma_{t}^{(1)}}}^{(1)}=-\frac{M}{2}\left(u^{2}+w^{2}+v_{E}^{2}\right),
\end{aligned}
$$




$$
\begin{aligned}
& \overline{\Gamma_{i}^{(2)}}=-\frac{M w}{2} \epsilon_{i j k} a^{j} \psi^{k}, \\
& \bar{\Gamma}_{u}^{(2)}=\overline{\Gamma_{w}^{(2)}}=0, \\
& \bar{\Gamma}_{\theta}^{(2)}=-\frac{M w^{2}}{2 \Omega}, \\
& \bar{\Gamma}_{t}^{(2)}=-\frac{M w}{2 \Omega} \mathbf{a} \cdot \nabla v_{E}^{2}-\frac{M w^{2}}{2 \Omega}\left(a v_{E} c\right)-\frac{m w}{\Omega}\left(\mathbf{a} \cdot \mathbf{v}_{E, t}\right), \\
& \overline{\Gamma_{i}^{(3)}}=-\frac{M w^{2}}{2 \Omega^{2}} \mathbf{a} \cdot \nabla(\mathbf{a} \times \boldsymbol{\psi})_{i}, \\
& \bar{\Gamma}_{u}^{(3)}=\bar{\Gamma}_{w}^{(3)}=0, \\
& \bar{\Gamma}_{\theta}^{(3)}=\frac{M w^{2}}{2 \Omega^{2}} \mathbf{b} \cdot \boldsymbol{\psi}, \\
& \overline{\Gamma_{t}^{(3)}}=-\frac{M w^{2}}{4 \Omega^{2}} \mathbf{a} \cdot \nabla\left(\hat{\mathbf{a}} \cdot \nabla v_{E}^{2}\right)-\frac{M w^{3}}{3 \Omega^{2}} \mathbf{a} \cdot \nabla\left(a v_{e} c\right) \\
& -\frac{M w^{2}}{2 \Omega^{2}} \mathbf{a} \cdot \nabla\left(\mathbf{a} \cdot \mathbf{v}_{E, t}\right), \\
& \overline{\Gamma_{i}^{(4)}}=-\frac{M w^{3}}{6 \Omega^{3}} \epsilon_{i j k} a^{j} a^{l} a^{m} \psi_{, l, m}^{k}, \\
& \bar{\Gamma}_{u}^{(4)}=\bar{\Gamma}_{w}^{(4)}=0, \\
& \bar{\Gamma}_{\theta}^{(4)}=\frac{M w^{3}}{3 \Omega^{3}}(a \psi b), \\
& \overline{\Gamma_{t}^{(4)}} \text { : is not needed. }
\end{aligned}
$$

We see that this preparatory transformation moves all dependence on gyroangle to $\mathscr{Q}\left(\epsilon^{2}\right)$ and higher in the Lagrangian 1 -forms.

With the preparatory transformation accomplished we return to the task of determining the transformation vector fields $g_{1}, g_{2}, \ldots$, and gauge functions $F^{(0)}, F^{(1)}, F^{(2)}, F^{(3)}, F^{(4)}, \ldots$, so as to eliminate any dependence on gyrophase from the Lagrangian 1-forms and determine the GC transformation to $\mathscr{O}\left(\epsilon^{2}\right)$. We do not alter the time coordinate and consequently none of the vector fields $g_{1}, g_{2}, \ldots$, have a time component.

A number of results relating products of various sorts with the vectors $\hat{\mathbf{a}}$ and $\hat{\mathbf{c}}$ are useful. They are analogous to $\partial \hat{\mathbf{a}} / \partial \theta=-\hat{\mathbf{c}}$ and $\partial \hat{\mathbf{c}} / \partial \theta=\hat{\mathbf{a}}$ in that these identities are useful for relating quantities involving $\hat{\mathbf{a}}$ and/or $\hat{\mathbf{c}}$ to derivatives with respect to gyroangle ( $\theta$ or $\Theta$ ). For arbitrary vectors $\boldsymbol{\alpha}$ and $\boldsymbol{\beta}$,

$$
\begin{aligned}
& (a \alpha a)+(c \alpha c)=\nabla \cdot \boldsymbol{\alpha}-(b \alpha b), \\
& (c \alpha a)=(a \alpha c)+\hat{\mathbf{b}} \cdot(\nabla \times \boldsymbol{\alpha}),
\end{aligned}
$$$$
(\hat{\mathbf{a}} \cdot \boldsymbol{\alpha})(\hat{\mathbf{a}} \cdot \boldsymbol{\beta})+(\hat{\mathbf{c}} \cdot \boldsymbol{\alpha})(\hat{\mathbf{c}} \cdot \boldsymbol{\beta})=\boldsymbol{\alpha} \cdot \boldsymbol{\beta}-(\hat{\mathbf{b}} \cdot \boldsymbol{\alpha})(\hat{\mathbf{b}} \cdot \boldsymbol{\beta}),
$$

$(\hat{\mathbf{a}} \cdot \boldsymbol{\alpha})(\hat{\mathbf{c}} \cdot \boldsymbol{\beta})-(\hat{\mathbf{c}} \cdot \boldsymbol{\alpha})(\hat{\mathbf{a}} \cdot \boldsymbol{\beta})=\hat{\mathbf{b}} \cdot(\boldsymbol{\beta} \times \boldsymbol{\alpha})$,

$(a \alpha c)(\hat{\mathbf{c}} \cdot \boldsymbol{\beta})+(a \alpha a)(\hat{\mathbf{a}} \cdot \boldsymbol{\beta})=(a \alpha \beta)-(\hat{\mathbf{b}} \cdot \boldsymbol{\beta})(a \alpha b)$,

$$
(a \alpha c)(\hat{\mathbf{a}} \cdot \boldsymbol{\beta})-(a \alpha a)(\hat{\mathbf{c}} \cdot \boldsymbol{\beta})=-\hat{\mathbf{b}} \cdot(\boldsymbol{\beta} \times \hat{\mathbf{a}} \cdot \nabla \boldsymbol{\alpha}) .
$$

These results are readily proved in Cartesian components and depend on the definitions of $\hat{\mathbf{a}}, \hat{\mathbf{b}}, \hat{\mathbf{c}}$ as given in Section II. As an example of the many ways these results are used we note that

$$
\left(a v_{e} c\right)=-\frac{1}{2} \hat{\mathbf{b}} \cdot \boldsymbol{\psi}-\frac{1}{2} \frac{\partial\left(a v_{e} a\right)}{\partial \Theta},
$$

which follows from Eq. (A32b).

To obtain the GC transformation to $\mathscr{Q}\left(\epsilon^{2}\right)$ and to eliminate the dependence on gyrophase in the phase space Lagrangian, we use the $\bar{\Gamma}$ from Eqs. (A24)-(A31) for the $\gamma$ in Eqs. (A6)-(A7). To achieve the results

$$
\begin{aligned}
& \Gamma_{\mathbf{X}}^{(0)}=\frac{M \Omega}{2} \hat{\mathbf{b}} \times \mathbf{X}, \\
& \Gamma_{U}^{(0)}=\Gamma_{W}^{(0)}=\Gamma_{\Theta}^{(0)}=0, \\
& \Gamma_{t}^{(0)}=-q \Phi(\mathbf{X}, t), \\
& \Gamma_{\mathbf{X}}^{(1)}=M U \hat{\mathbf{b}}+M \mathbf{v}_{e}, \\
& \Gamma_{U}^{(1)}=\Gamma_{W}^{(1)}=\Gamma_{\Theta}^{(1)}=0, \\
& \Gamma_{t}^{(1)}=-\frac{M}{2}\left(U^{2}+W^{2}+v_{e}^{2}\right), \\
& \Gamma_{\mathbf{X}}^{(2)}=0, \\
& \Gamma_{U}^{(2)}=\Gamma_{W}^{(2)}=0, \\
& \Gamma_{\Theta}^{(2)}=-\frac{M W^{2}}{2 \Omega}, \\
& \Gamma_{t}^{(2)}=-\frac{M W^{2}}{4 \Omega} \hat{\mathbf{b}} \cdot \psi
\end{aligned}
$$

we make the following choices:

$$
\begin{aligned}
& \mathbf{g}_{1}=0, \quad F^{(0)}=0, \quad F^{(1)}=0, \\
& \mathbf{g}_{2}=\frac{W}{\Omega^{2}}[(\hat{\mathbf{b}} \cdot \boldsymbol{\psi}) \hat{\mathbf{a}}+(\hat{\mathbf{a}} \cdot \boldsymbol{\psi}) \hat{\mathbf{b}}], \\
& g_{1}^{U}=\frac{W}{\Omega} \hat{\mathbf{c}} \cdot \boldsymbol{\psi}, \\
& g_{1}^{W}=\frac{W}{\Omega}\left[\frac{1}{2}\left(a v_{e} c\right)-\frac{1}{4}(\hat{\mathbf{b}} \cdot \boldsymbol{\psi})\right]+\frac{1}{\Omega} \hat{\mathbf{a}} \cdot\left(\frac{D \mathbf{v}_{e}}{D t}+v_{\|} \boldsymbol{\psi}\right), \\
& g_{1}^{\Theta}=\frac{1}{2 \Omega}\left(a v_{e} a\right)-\frac{1}{W \Omega} \hat{\mathbf{c}} \cdot\left(\frac{D \mathbf{v}_{e}}{D t}+v_{\|} \boldsymbol{\psi}\right), \\
& F^{(3)}=\frac{M W^{2}}{4 \Omega^{2}}\left(a v_{e} a\right)-\frac{M W}{\Omega^{2}} \hat{\mathbf{c}} \cdot\left(\frac{D \mathbf{v}_{e}}{D t}+v_{\|} \boldsymbol{\psi}\right) .
\end{aligned}
$$

The procedure followed to carry this transformation to one more level is the same. We choose the appropriate components of $g_{2}, g_{3}$ and the gauge function $F^{(4)}$ to achieve 


$$
\Gamma_{\mathbf{X}}^{(3)}=0, \quad \Gamma_{U}^{(3)}=0, \quad \Gamma_{W}^{(3)}=0, \quad \Gamma_{\Theta}^{(3)}=0,
$$

and force all corrections into $\Gamma_{t}^{(3)}$ with no dependence on the gyrophase $\Theta$. The algebra is tedious and the results lengthy.
In arriving at these results in the forms given we have made frequent use of Eqs. (A32). We use the notation $g_{3}^{a}, g_{3}^{b}, g_{3}^{c}$ to denote the components of $\mathbf{g}_{3}$ along the directions $\hat{\mathbf{a}}, \mathbf{b}, \hat{\mathbf{c}}$. The lengthy expression for $F^{(4)}$ is available from the author upon request.

$$
\begin{aligned}
& g_{3}^{a}=\frac{W^{2}}{\Omega^{3}}\left[\frac{1}{2}(a \psi b)-\frac{1}{4} \hat{\mathbf{c}} \cdot \nabla\left(a v_{e} a\right)\right]+\frac{W}{\Omega^{3}}\left[(\hat{\mathbf{a}} \cdot \boldsymbol{\psi})^{2}-\frac{3}{4}(\hat{\mathbf{b}} \cdot \boldsymbol{\psi})^{2}-\frac{1}{2}\left(a v_{e} c\right)(\hat{\mathbf{b}} \cdot \boldsymbol{\psi})+\left(c \frac{D v_{e}}{D t} c\right)\right] \\
& +\frac{W v_{\|}}{\Omega^{3}}(c \psi c)-\frac{1}{\Omega^{3}}\left[(\hat{\mathbf{b}} \cdot \psi)\left(\hat{\mathbf{a}} \cdot \frac{D \mathbf{v}_{e}}{D t}\right)\right]-\frac{v_{\|}}{\Omega^{3}}(\hat{\mathbf{a}} \cdot \psi)(\hat{\mathbf{b}} \cdot \psi), \\
& g_{3}^{b}=\frac{W^{2}}{16 \Omega^{3}}\left[(b \psi b)+4(a \psi a)-2 \hat{\mathbf{b}} \cdot \nabla\left(a v_{e} c\right)\right]-\frac{3 W v_{\|}}{\Omega^{3}}(b \psi a)+\frac{W}{\Omega^{3}}\left[\frac{3}{4}\left(c v_{e} \psi\right)+\frac{1}{4} \hat{\mathbf{b}} \cdot\left(\boldsymbol{\psi} \times \hat{\mathbf{a}} \cdot \nabla \mathbf{v}_{e}\right)-\frac{3}{2}(\hat{\mathbf{a}} \cdot \boldsymbol{\psi})(\hat{\mathbf{b}} \cdot \boldsymbol{\psi})\right. \\
& \left.-\left(b \frac{D v_{e}}{D t} a\right)+\left(\hat{\mathbf{c}} \cdot \frac{D \psi}{D t}\right)-(\hat{\mathbf{a}} \cdot \boldsymbol{\psi})\left(\hat{\mathbf{b}} \cdot \nabla v_{\|}\right)\right]-\frac{v_{\|}}{2 \Omega^{3}}\left[\psi^{2}-(\hat{\mathbf{b}} \cdot \boldsymbol{\psi})^{2}\right]-\frac{1}{2 \Omega^{3}}\left[\psi \cdot \frac{D \mathbf{v}_{e}}{D t}-(\hat{\mathbf{b}} \cdot \boldsymbol{\psi}) \hat{\mathbf{b}} \cdot \frac{D \mathbf{v}_{e}}{D t}\right], \\
& g_{3}^{c}=\frac{W^{2}}{4 \Omega^{3}} \hat{\mathbf{c}} \cdot \nabla\left(a v_{e} c\right)+\frac{W}{\Omega^{3}}\left[\frac{1}{2}(\hat{\mathbf{b}} \cdot \boldsymbol{\psi})\left(a v_{e} a\right)+(\hat{\mathbf{c}} \cdot \boldsymbol{\psi})(\hat{\mathbf{a}} \cdot \boldsymbol{\psi})-\left(c \frac{D \mathbf{v}_{e}}{D t} c\right)\right]-\frac{W v_{\|}}{\Omega^{3}}(c \psi c)-\frac{1}{\Omega^{3}}(\hat{\mathbf{b}} \cdot \boldsymbol{\psi}) \hat{\mathbf{c}} \cdot \frac{D \mathbf{v}_{e}}{D t}-\frac{v_{\|}}{\Omega^{3}}(\hat{\mathbf{c}} \cdot \boldsymbol{\psi}) \\
& \times(\hat{\mathbf{b}} \cdot \psi), \\
& g_{2}^{U}=\frac{W^{2}}{4 \Omega^{2}}\left[\hat{\mathbf{b}} \cdot \nabla\left(a v_{e} a\right)+2(a \psi c)\right]-\frac{W}{\Omega^{2}}\left[\left(b \frac{D v_{e}}{D t} c\right)+\frac{1}{4}\left(a v_{e} \psi\right)+\frac{7}{8}(\hat{\mathbf{c}} \cdot \boldsymbol{\psi})(\hat{\mathbf{b}} \cdot \boldsymbol{\psi})\right]-\frac{W v_{\|}}{\Omega^{2}}(b \psi c)+\frac{1}{2 \Omega^{2}} \hat{\mathbf{b}} \cdot\left(\frac{D \mathbf{v}_{e}}{D t} \times \boldsymbol{\psi}\right), \\
& g_{2}^{W}=\frac{1}{2 \Omega}(\hat{\mathbf{b}} \cdot \boldsymbol{\psi}) g_{1}^{W}+\frac{W}{2 \Omega^{2}}\left[(\hat{\mathbf{b}} \cdot \boldsymbol{\psi})^{2}-(\hat{\mathbf{c}} \cdot \boldsymbol{\psi})^{2}\right]-\frac{W^{2}}{3 \Omega^{2}}(a \psi b)-\frac{\Omega}{M W} \frac{\partial F^{(4)}}{\partial \Theta}, \\
& g_{2}^{\Theta}=\frac{1}{2 \Omega}(\hat{\mathbf{b}} \cdot \boldsymbol{\psi}) g_{1}^{\Theta}-\frac{1}{2 \Omega^{2}}(\hat{\mathbf{a}} \cdot \boldsymbol{\psi})(\hat{\mathbf{c}} \cdot \boldsymbol{\psi})+\frac{\Omega}{M W} \frac{\partial F^{(4)}}{\partial W} .
\end{aligned}
$$

Using the definitions given in Eq. (5) and Eq. (4) we find for $\Gamma_{t}^{(3)}$ the result given in Eq. (3). From this point on the pattern is the same. We select the equations

$$
\Gamma_{\mathbf{X}}^{(k)}=\Gamma_{U}^{(k)}=\Gamma_{W}^{(k)}=\Gamma_{\Theta}^{(k)}=0 \quad \text { and } \frac{\partial \Gamma_{t}^{(k)}}{\partial \Theta}=0
$$

to determine the generating vector fields $g_{k}$ and gauge functions $F^{(k)}$ while pushing all corrections into the Hamiltonian function $\Gamma_{t}^{(k)}$.

With the GC transformation fully determined to the order needed, we turn our attention to the functions required for the density in Eq. (A18). The Jacobian of the transformation $J \equiv M^{3} \partial(\cdots) / \partial(\cdots)$ is easily computed from the components of the Lagrangian 1-forms. Using

$$
\omega_{\mu \nu}=\frac{\partial \Gamma_{\nu}}{\partial Z^{\mu}}-\frac{\partial \Gamma_{\mu}}{\partial Z^{\nu}}, \quad \mu, \nu=1,2, \ldots, 6 \quad \text { and } \operatorname{det}(\omega)=J^{2},
$$

we find

$$
J(\mathbf{X}, W, t)=M^{3} W\left(1+\frac{\epsilon}{\Omega}(\hat{\mathbf{b}} \cdot \boldsymbol{\psi})\right),
$$

accurate to all orders.
For $F$ in Eq. (A20) we substitute

$$
F+\epsilon g_{p}^{\mu} \frac{\partial F}{\partial Z^{\mu}}+\frac{1}{2} \epsilon^{2} g_{p}^{\nu} \frac{\partial}{\partial Z^{\nu}}\left(g_{p}^{\mu} \frac{\partial F}{\partial Z^{\mu}}\right),
$$

obtained from the preparatory transformation $g_{p}$ of Eq. (A23). Keeping terms only to $\mathscr{Q}\left(\epsilon^{2}\right)$ we find

$$
T F=F+\epsilon \cdot \mathscr{b}_{1}+\epsilon^{2} \cdot \mathscr{b}_{2}+\mathscr{Q}\left(\epsilon^{3}\right),
$$

where

$$
\mathscr{C}_{1}=\left(g_{p}^{\mu}+g_{1}^{\mu}\right) \frac{\partial F}{\partial Z^{\mu}}
$$

and

$$
\begin{aligned}
\mathscr{C}_{2}= & g_{2}^{\mu} \frac{\partial F}{\partial Z^{\mu}}+\frac{1}{2} g_{1}^{\nu} \frac{\partial}{\partial Z^{\nu}}\left(g_{1}^{\mu} \frac{\partial F}{\partial Z^{\mu}}\right)+\frac{1}{2} g_{p}^{\nu} \frac{\partial}{\partial Z^{\nu}}\left(g_{p}^{\mu} \frac{\partial F}{\partial Z^{\mu}}\right) \\
& +g_{1}^{\nu} \frac{\partial}{\partial Z^{\nu}}\left(g_{p}^{\mu} \frac{\partial F}{\partial Z^{\mu}}\right) .
\end{aligned}
$$

With the results obtained for the generating vector fields given in Eqs. (A37) and the fact the $F$ does not depend on $\Theta$, we find 


$$
\begin{aligned}
& \mathscr{C}_{1}(\mathbf{X}, U, \Theta, W, t)=-\frac{W}{\Omega} \hat{\mathbf{a}} \cdot \nabla F+g_{1}^{U} F,_{U}+g_{1}^{W} F, W, \\
& \mathscr{b}_{2}(\mathbf{X}, U, \Theta, W, t)=\mathbf{g}_{2} \cdot \nabla F-\frac{1}{\Omega} g_{1}^{W} \hat{\mathbf{a}} \cdot \nabla F+\frac{W}{\Omega} g_{1}^{\Theta} \hat{\mathbf{c}} \cdot \nabla F+\frac{1}{2} F,{ }_{U}\left(2 g_{2}^{U}+g_{1}^{U} g_{1}^{U},{ }_{U}+g_{1}^{\Theta} g_{1}^{U},{ }_{\Theta}+g_{1}^{W} g_{1}^{U},{ }_{W}\right)+\frac{1}{2} F,{ }_{W}\left(2 g_{2}^{W}\right. \\
& \left.+g_{1}^{U} g_{1}^{W},{ }_{U}+g_{1}^{\Theta} g_{1}^{W},{ }_{\Theta}+g_{1}^{W} g_{1}^{W},{ }_{W}\right)+\frac{1}{2}\left(g_{1}^{U}\right)^{2} F,{ }_{U U}+\frac{1}{2}\left(g_{1}^{W}\right)^{2} F,{ }_{W W}+g_{1}^{U} g_{1}^{W} F,{ }_{U W}+\frac{1}{2} \frac{W^{2}}{\Omega^{2}} \hat{\mathbf{a}} \cdot \nabla(\hat{\mathbf{a}} \cdot \nabla F) \\
& -\frac{W}{\Omega} g_{1}^{U} \hat{\mathbf{a}} \cdot \nabla F,{ }_{U}-\frac{W}{\Omega} g_{1}^{W} \hat{\mathbf{a}} \cdot \nabla F, W
\end{aligned}
$$

The Dirac delta function in Eq. (A18) evaluates $\mathbf{x}^{\prime}$ at $\mathbf{x}$, where from Eq. (A16) we find

$$
\mathbf{X}^{\prime}=\mathbf{X}+\frac{\epsilon W}{\Omega} \hat{\mathbf{a}}-\frac{\epsilon^{2} W}{\Omega^{2}}[(\hat{\mathbf{b}} \cdot \psi) \hat{\mathbf{a}}+(\hat{\mathbf{a}} \cdot \psi) \hat{\mathbf{b}}] .
$$

Writing $\mathbf{X}=\mathbf{x}+\Delta \mathbf{x}$ we must then expand $T F$ of Eq. (A43) about $\mathbf{x}$ where

$$
\Delta \mathbf{x}=-\frac{\epsilon W}{\Omega} \hat{\mathbf{a}}+\frac{\epsilon^{2} W}{\Omega^{2}}[(\hat{\mathbf{b}} \cdot \psi) \hat{\mathbf{a}}+(\hat{\mathbf{a}} \cdot \psi) \hat{\mathbf{b}}]=\epsilon \mathbf{g}_{p}+\epsilon^{2} \mathbf{g}_{2} .
$$

Note that the $\mathscr{O}\left(\epsilon^{2}\right)$ term in Eq. (A48) can be written immediately as a function of $\mathbf{x}$ or $\mathbf{X}$; the $\mathcal{Q}(\epsilon)$ term is not a function of the spatial coordinates. Thus in $T F$ of Eq. (A43), $F$ must be expanded to second order, $\mathscr{b}_{1}$ to first order, and $\mathscr{b}_{2}$ to zero order. Then we write

$$
T F=F+\epsilon \overline{\mathscr{b}}_{1}+\epsilon^{2} \overline{\mathscr{b}}_{2}+\mathscr{Q}\left(\epsilon^{3}\right) \text {, }
$$

where

$$
\begin{aligned}
\overline{\mathscr{C}}_{1}(\mathbf{x}, U, \Theta, W, t)= & \mathscr{C}_{1}-\frac{W}{\Omega} \hat{\mathbf{a}} \cdot \nabla F, \\
\overline{\mathscr{C}}_{2}(\mathbf{x}, U, \Theta, W, t)= & \mathscr{C}_{2}+\mathbf{g}_{2} \cdot \nabla F+\frac{3 W^{2}}{2 \Omega^{2}} \hat{\mathbf{a}} \cdot \nabla(\hat{\mathbf{a}} \cdot \nabla \mathbf{F}) \\
& -\frac{W}{\Omega} \hat{\mathbf{a}} \cdot \nabla\left(g_{1}^{U} F,,_{U}+g_{1}^{W} F,{ }_{W}\right),
\end{aligned}
$$

with the functions on the right-hand sides depending on $\mathbf{x}$ and not $\mathbf{X}$. Similarly the Dirac delta function requires the expansion of the Jacobian of Eq. (A41),

$$
\begin{aligned}
J(\mathbf{x}, W, t)= & M^{3} W\left(1+\frac{\epsilon}{\Omega}(\hat{\mathbf{b}} \cdot \psi)-\frac{\epsilon^{2} W}{\Omega^{2}} \hat{\mathbf{a}} \cdot \nabla(\hat{\mathbf{b}} \cdot \psi)\right) \\
& +\mathscr{Q}\left(\epsilon^{3}\right) .
\end{aligned}
$$

Substituting these results into Eq. (A18) gives the particle density at the field point $\mathbf{x}$,

$$
\begin{aligned}
n(\mathbf{x}, t)= & M^{3} \int\left[F+\epsilon\left\{\frac{1}{\Omega}(\hat{\mathbf{b}} \cdot \boldsymbol{\psi}) F+\overline{\mathscr{C}}_{1}\right\}\right. \\
& +\epsilon^{2}\left\{-\frac{W}{\Omega^{2}} \hat{\mathbf{a}} \cdot \nabla(\hat{\mathbf{b}} \cdot \boldsymbol{\psi}) F+\frac{1}{\Omega}(\hat{\mathbf{b}} \cdot \boldsymbol{\psi}) \overline{\mathscr{C}}_{1}\right. \\
& \left.\left.+\overline{\mathscr{C}}_{2}\right\}\right] W d W d U d \Theta .
\end{aligned}
$$

The integral over $\Theta$ kills all terms that are of odd order in $\hat{\mathbf{a}}$ and $\hat{\mathbf{c}}$. Only when we have terms even in these vectors can we get something other than zero. For example, we find

$$
\int_{0}^{2 \pi} \overline{\mathscr{C}}_{1} d \Theta=-\frac{\pi W}{\Omega}(\hat{\mathbf{b}} \cdot \boldsymbol{\psi}) F, W .
$$

Carrying out the integrations over angle in Eq. (A55) results in Eq. (21) for the density.

${ }^{1}$ C. F. Driscoll, Phys. Rev. Lett. 64, 645 (1990).

${ }^{2}$ R. A. Smith and M. N. Rosenbluth, Phys. Rev. Lett. 64, 649 (1990).

${ }^{3}$ R. A. Smith, Phys. Fluids B 4, 287 (1992).

${ }^{4}$ S. N. Rasband, R. L. Spencer, and R. R. Vanfleet, Phys. Fluids B 5, 669 (1993).

${ }^{5}$ R. G. Littlejohn, J. Plasma Phys. 29, 111 (1983).

${ }^{6}$ A. J. Brizard, Phys. Plasmas 2, 459 (1995).

${ }^{7}$ A. Brizard, J. Plasma Phys. 41, 541 (1989).

${ }^{8}$ T. S. Hahm, W. W. Lee, and A. Brizard, Phys. Fluids 31, 1940 (1988).

${ }^{9}$ T. S. Hahm, Phys. Fluids 31, 2670 (1988).

${ }^{10}$ H. Ye and A. N. Kaufman, Phys. Fluids B 4, 1735 (1992).

${ }^{11}$ D. H. D. Dubin, J. A. Krommes, C. Oberman, and W. W. Lee, Phys. Fluids 26, 3524 (1983).

${ }^{12}$ D. R. Nicholson, Introduction to Plasma Theory (Wiley, New York, 1983), Chap. 6.

${ }^{13}$ R. J. Briggs, J. D. Daugherty, and R. H. Levy, Phys. Fluids 13, 421 (1970).

${ }^{14}$ M. Abramowitz and I. Stegun, Handbook of Mathematical Functions (Dover, New York, 1972), Chap. 5. 